\title{
Nerve sheath myxoma of the lower lid
}

\author{
Yu Nakamura, Toshitaka Hirakata, Makio Mukai
}

Nerve sheath myxoma is a rare benign tumour that principally occurs in the region of the head and neck. There are only two cases reported in the field of ophthalmology to our knowledge. ${ }^{12}$ We recently managed a case of a chalazion-like nerve sheath myxoma of the lower eyelid.

\section{Case report}

A 23-year-old woman complained of a painless tumour on the left lower eyelid near the punctum for 5 months. The initial diagnosis was chalazion, and local treatment with antibiotic and corticosteroid eyedrops was performed.

She visited our clinic on 27 July 1990, because the tumour had gradually increased to $5 \mathrm{~mm}$ in diameter. We operated on the tumour on 25 September 1990 under local anaesthesia, but it could not be totally resected because of the proximity to the punctum (Fig 1). Regrowth was observed 4 months later and a second operation was performed on 28 June 1991. After the second operation, no regrowth of the tumour was observed for 18 months.

Macroscopically, the tumour was reddish and multinodular.

Histopathologically it was not encapsulated and located widely from the subepidermal tissue

Department of
Ophthalmology, Keio
University, School of
Medicine, Tokyo, Japan
Y Nakamura
T Hirakata
Division of Surgical
Pathology, Central
Clinical Laboratories,
Keio University Hospital
M Mukai
Correspondence to:
Yu Nakamura, MD, Keio
University Hospital,
Department of
Ophthalmology, 35
Shinanomachi, Shinjuku-ku,
Tokyo, Japan.
Accepted for publication
1l April 1994

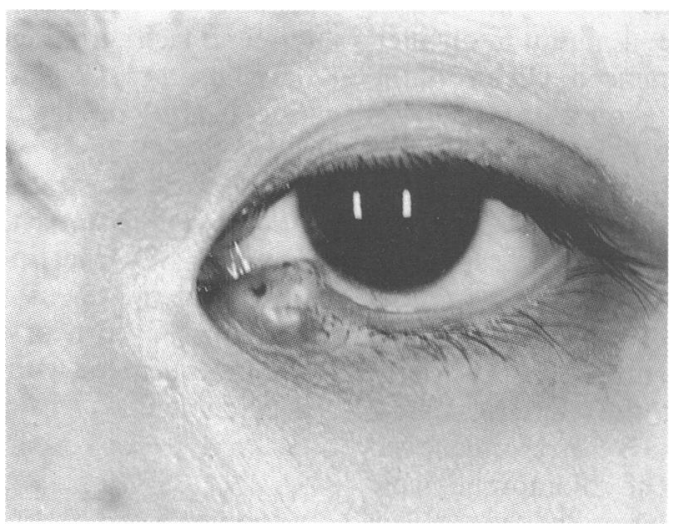

Figure 1 Photograph of patient's eye on first visit. Tumour of the left lower eyelid is observed.

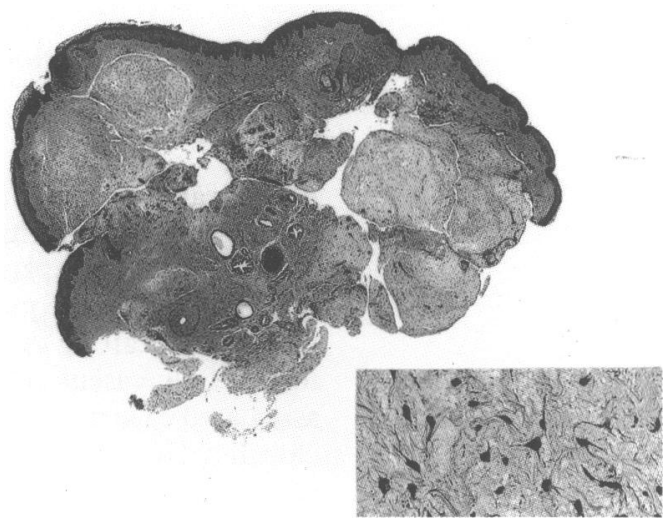

Figure 2 The tumour presented a multinodular pattern with markedly myxomatous changes (haematoxylin and eosin staining, $\times 14)$. Inset: Nodules with myxomatous change composed of stellate and spindle cells $(\times 186)$.

to the deep connective tissue. Stellate or spindeshaped tumour cells composed of multiple nodules with markedly myxomatous changes (Fig 2). Cells were positive for S-100 protein by immunohistochemical staining, and the stroma of the tumour was positive for Alcian blue, but negative for periodic acid Schiff and mucicarmine staining. There were no findings of reticulum fibre proliferation.

\section{Comment}

According to Enzinger's classification of peripheral nerve the tumours proposed in 1983, tumours with markedly myxomatous and multinodular pattern are classified as nerve sheath myxomas. ${ }^{3}$ Tumour cells are believed to originate from fibroblasts or Schwann cells located in the perineural layer. In our case, tumour cells were positive for S-100 protein, suggesting that the tumour was derived from Schwann cells.

1 Hirata A, Maruoka S. Nerve sheath myxoma of the lid - report of a case. Folia Ophthalmol fap 1992; 43: 95-104.

2 Stringa M, Carnevali L, Castello A. Neurothekeoma of the eyelid report of a case. Ann Ottalmol Clin Ocul 1988; 959-64.

3 Enzinger FM, Weiss SW. Soft tissue tumors. 2nd ed. St Louis: Mosby, 1988; 6-8: 768-9. 